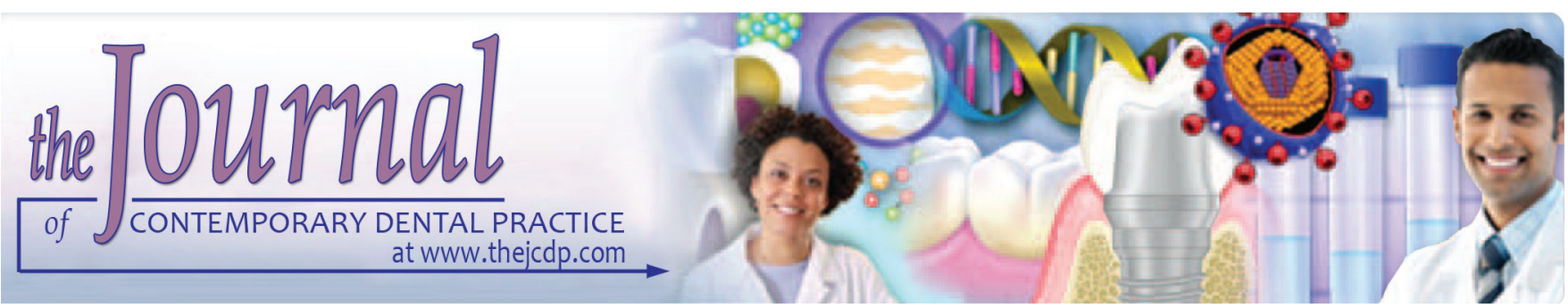

\title{
In vitro Analysis of Shear Bond Strength and Adhesive Remnant Index of Stainless Steel Brackets with Different Adhesive Systems to Enamel
}

${ }^{1}$ Nishad A Vaheed, ${ }^{2}$ Mukesh Gupta, ${ }^{3}$ Sumitha A David, ${ }^{4}$ George Sam, ${ }^{5}$ Pavithra K Ramanna, ${ }^{6}$ Sudeep C Bhagvandas

\begin{abstract}
Aim: This study aimed at evaluating and comparing the bond strength of stainless steel brackets bonded with multiple orthodontic adhesives.

Materials and methods: About 60 premolars extracted to create space during orthodontic treatment were included in the present study. Premolar stainless steel brackets of size 0.022 slot with the surface area of bracket base of $11.15 \mathrm{~mm}^{2}$ were used. The premolars were divided randomly into three groups of 20 in each. Group I: Conventional Acid Etching (Transbond $\mathrm{XT}$ ); group II: Seventh-generation adhesive materials (Xeno V); and group III: Flowable composite (Filtek Z350 XT). Calculation of Adhesive Remnant Index (ARI) was done and shear bond strength was assessed. The probability level of 0.05 was considered as clinically significant.
\end{abstract}

Results: The shear bond strength was highest in group II (17.46 \pm 1.36$)$, which was followed by group I $(15.33 \pm 2.78)$ and

\footnotetext{
${ }^{1}$ Department of Orthodontics and Dentofacial Orthopedics Noorul Islam College of Dental Science, Thiruvananthapuram Kerala, India

${ }^{2}$ Department of Orthodontics and Dentofacial Orthopedics Index Institute of Dental Sciences, Indore, Madhya Pradesh India

${ }^{3}$ Department of Orthodontics and Dentofacial Orthopedics Educare Institute of Dental Sciences, Malappuram, Kerala, India

${ }^{4}$ Department of Preventive Dental Sciences, College of Dentistry Prince Sattam Bin Abdulaziz University, Al kharj, Kingdom of Saudi Arabia

${ }^{5}$ Department of Prosthodontics and Crown and Bridge, Vydehi Institute of Dental Sciences and Research Centre, Bengaluru Karnataka, India

${ }^{6}$ Department of Public Health Dentistry, Sree Anjaneya Institute of Dental Sciences, Kozhikode, Kerala, India

Corresponding Author: Nishad A Vaheed, Department of Orthodontics and Dentofacial Orthopedics, Noorul Islam College of Dental Science, Thiruvananthapuram, Kerala, India, e-mail: drnishath@hotmail.com
}

the least was shown by group III $(13.96 \pm 1.44)$. A statistically significant difference was found between group I vs II, group II vs III, and group III vs II. Utmost prevalence of about 35\% was found in Transbond XT with an ARI score of 3 (followed by all adhesive left on the tooth), Filtek Z350 XT with $20 \%$, and the least was found with Xeno $\mathrm{V}$, about $5 \%$. The probability was found to be $p=0.025$ among different groups, which is statistically significant according to chi-square test.

Conclusion: The study concluded that the adhesive materials of the seventh generation showed higher shear bond strength than that of the flowable composite and conventional acid etching. The ARI indicated that a minimum amount of the residual adhesive was found on the surface of the enamel after debonding.

Clinical significance: This study helps orthodontists to select appropriate adhesive and orthodontic brackets for the benefit of the patient and to distinguish the distribution of stress homogeneously within the layer of cement while loading and subsequently minimizing the damage to the enamel during the debonding of orthodontic brackets.

Keywords: Adhesive, Adhesive remnant index, Brackets, Shear bond strength.

How to cite this article: Vaheed NA, Gupta M, David SA, Sam G, Ramanna PK, Bhagvandas SC. In vitro Analysis of Shear Bond Strength and Adhesive Remnant Index of Stainless Steel Brackets with Different Adhesive Systems to Enamel. J Contemp Dent Pract 2018;19(9):1047-1051.

Source of support: Nil

Conflict of interest: None

\section{INTRODUCTION}

Orthodontics, a specialty of dentistry, in recent years, is concerned more with esthetics. The specialty has shown evolution in its esthetic ideologies, and with the materials which are commonly used. The patients are concerned not only with their smile and appearance but also with the appliances used and their appearance. Demand for 
the esthetic appliances has increased with increase in the adults who are seeking orthodontic treatment. ${ }^{1}$

For successful orthodontic treatment, the clinical behavior of the orthodontic brackets and the strength of the bond play a major role. ${ }^{2}$ The materials used during the manufacture of the orthodontic brackets are: Plastic, stainless steel, ceramics, and titanium. ${ }^{3}$

Buonocore $^{4}$ introduced enamel-etching technique because of which orthodontic accessories' direct bonding to welded metal bands became possible. The studies were conducted over years of direct bonding of brackets to enamel. In an attempt to improve the bonding strength in orthodontics, evaluation of the different types of bonding systems was done and surface preparation of enamel was conducted. ${ }^{5}$

A variety of orthodontic bonding and adhesive techniques are developed and are subjected to various in vitro and in vivo studies. The individual base of the orthodontic attachment (material and mechanical properties), tooth surface (enamel preparation and morphology), and the bonding agent itself (material composition and shear bond strength) are the three main components which must be considered for successful orthodontic bonding. ${ }^{6}$ The wide range of cement materials, such as chemicalcured, differently filled resins, light-activated, and other materials are available for orthodontists. The main aim is to attain adequate marginal seal and minimum utilization of the bonding material for bonding the brackets, especially to avoid white spot lesions and dental caries below and around the brackets. ${ }^{7}$

There is an evolution through many generations of dental adhesive along with a change in mechanism, chemistry, application technique, clinical effectiveness, and the number of bottles. In the latest bonding system generation, the trend is to reduce the clinical steps for placement and components count. The single-bottle adhesive system as an introduction for new-generation material contains a combination of desensitizer, adhesive, and etchant as one component. ${ }^{8}$ The main aim of this study is to compare and evaluate the debonding strength of the orthodontic adhesives which are bonded with stainless steel brackets.

\section{MATERIALS AND METHODS}

\section{Sample Selection and Selection of Teeth}

The sample size was calculated using the below formula: ${ }^{9}$

n-Sample size

$$
\mathrm{n}=\frac{2\left(Z_{\mathrm{a}}+\mathrm{Z}_{1-\beta}\right)^{2^{2}{ }^{2}}}{\Delta^{2}}
$$

$$
\begin{gathered}
Z_{a}-1.96 \\
Z_{1-\beta}-0.84 \\
\Delta^{2}-(\text { Effect size })^{2}
\end{gathered}
$$

The sample size for the present study was 60 .

Sixty premolars extracted to create space during orthodontic treatment were collected. The extracted teeth were stored to avoid bacterial growth in $0.1 \%(\mathrm{w} / \mathrm{v})$ thymol. The teeth were with an absence of cracks, previously treated with chemical agents (hydrogen peroxide), caries, with intact buccal enamel, and extracted maximum before 3 months of use.

\section{Selection of Brackets}

Premolar stainless steel brackets with 0.022 " slot and bracket base of $11.15 \mathrm{~mm}^{2}$ were used.

\section{Bonding Procedure}

Sixty premolars were divided randomly into three groups as mentioned below:

\section{Group I: Conventional Acid Etching (Transbond XT)}

The surface of the teeth was etched using 37\% phosphoric acid for 15 seconds, washed using water, and dried till chalky white appearance. The adhesive primer on the surface of etched enamel was applied; orthodontic bracket was placed and bonded using Transbond XT (3M Unitek, California, USA).

\section{Group II: Seventh-generation Bonding Agent (Xeno V)}

The conditioning of the teeth with self-etching primer seventh-generation light-cure (Xeno V, Dentsply, Konstanz, Germany) was done. It is a self-etching, light-curing, and one-component adhesive. As per the recommendation of the manufacturer, the material was used in two layers, photopolymerized for 15 seconds, air jet was lightly applied and light-cured for 10 seconds. The bonding is done using light-cured adhesive resin (Dentsply, Konstanz, Germany).

\section{Group III: Flowable Composite (Filtek Z350 XT)}

As per the instruction of the manufacturer, the primer (Adper adhesive systems; 3M ESPE, Seefeld, Germany) was applied in a thin layer over the etched surface and light-cured for 10 seconds. The bracket was placed using composite and cured for 20 seconds.

\section{Debonding of Stainless Brackets}

Occlusogingival load at the bracket-tooth interface was applied to create a shear force. The flat end of a steel rod which was attached to the crosshead of the universal testing machine (Instron Corp., Canton, Massachusetts, USA) was used to create the load. The tooth facial surface was kept parallel to the applied force while testing the shear bond strength using a mounting jig. The 
In vitro Analysis of Shear Bond Strength and Adhesive Remnant Index

measurement of bond strength was made at a crosshead speed of $1 \mathrm{~mm} / \mathrm{min}$, the load applied was recorded in Newton at the time of fracture, and finally, calculation was done by dividing debonding force by bracket base surface area measured by megapascals ( $\mathrm{MPa})$.

\section{Assessment of Adhesive Remnant Index}

The fractured specimen was examined after debonding and as per Artun and Bergland, ${ }^{10}$ ARI was determined.

- Score 0: No adhesive left on the tooth

- Score 1: Less than half of the adhesive left on the tooth

- Score 2: More than half of the adhesive left on the tooth

- Score 3: All adhesive left on the tooth, with a distinct impression of the bracket mesh.

To define the sites of failure of the bond between bracket base, resin, and enamel in more comprehensive means, ARI scores were used. Using an optical stereomicroscope with a magnification of $\times 40$, the ARI scores were assessed.

\section{Statistical Analysis}

Considering the two factors, type of bracket and loading mode and their interaction, the comparison of bond strength's mean values was done using a Tukey's multiple comparison tests and two-way analysis of variance (ANOVA). The probability level was set to be 0.05 for statistical significance. The difference in the ARI values was determined using the chi-square $\left(\chi^{2}\right)$ test.

\section{RESULTS}

The different adhesive materials' mean shear bond strength is shown in Table 1 . The highest shear bond strength was seen in group II (17.46 \pm 1.36$)$, followed by group I $(15.33 \pm 2.78)$, and the least bond strength was found in group III $(13.96 \pm 1.44)$. The analysis of variance showed high statistical difference within the adhesive materials.

The comparisons between the different adhesive materials are found in Table 2. The comparison showed a significant difference statistically between group I vs II, group II vs III, and group III vs II.

The individual ARI scores of the adhesive material in the three groups are mentioned in Table 3. The ARI score was 3 (all adhesive left on the tooth). The most prevalent was Transbond XT, about 35\%, followed by Filtek Z350 XT, about $20 \%$, and least was found with Xeno V, about

Table 1: Mean shear bond strength of different adhesive materials

\begin{tabular}{|c|c|c|c|c|c|}
\hline Groups & Mean & $S D$ & F-value & $p$-value & Significance \\
\hline I & 15.33 & 2.78 & 26.144 & 0.0001 & $\mathrm{HS}$ \\
\hline II & 17.46 & 1.36 & & & \\
\hline III & 13.96 & 1.44 & & & \\
\hline
\end{tabular}

Table 2: Multiple comparisons of different adhesive systems using Tukey's post hoc test

\begin{tabular}{llll}
\hline Groups & Compared with & Mean difference & Significance \\
\hline I & Group II & -2.13 & 0.04 \\
& Group III & 1.37 & 0.24 \\
II & Group I & 2.13 & 0.02 \\
& Group III & 3.5 & 0.001 \\
III & Group I & -1.37 & 0.08 \\
& Group II & -3.5 & 0.001 \\
\hline
\end{tabular}

Table 3: ARI scores of different adhesive materials

\begin{tabular}{llllll}
\hline Groups & $n$ & Score 0 & Score 1 & Score 2 & Score 3 \\
\hline I & 20 & $3(15 \%)$ & $5(25 \%)$ & $5(25 \%)$ & $7(35 \%)$ \\
II & 20 & $7(35 \%)$ & $8(40 \%)$ & $4(20 \%)$ & $1(5 \%)$ \\
III & 20 & $5(25 \%)$ & $7(35 \%)$ & $4(20 \%)$ & $4(20 \%)$ \\
\hline
\end{tabular}

Chi-square $\left(\chi^{2}\right)=16.42 ; p=0.024$

5\%. After comparing the ARI scores within the groups, the chi-square test showed a probability of 0.024 , which was statistically significant. This proved that the adhesive material left on the tooth was high in Transbond XT group compared with Filtek Z350 XT and Xeno V groups.

\section{DISCUSSION}

The direct bonding system has made a revolution in bonding the orthodontic brackets and has made advancement in the clinical practice for orthodontists. Acid etchants and primer are used as important parts of the bonding procedure nowadays, to allow the sealant to wet and penetrate to bond the brackets on enamel. The continued introduction of new materials with improvements minimizes the loss of enamel, reduces the chairside time, simplifies the bonding procedures, and makes it even much predictable. ${ }^{11}$

During removal of brackets, failure of the bond may occur within the adhesive, or at the bracket-adhesive interface or at the adhesive-enamel interface. Increase in the bond strength leads to the bond failure movement toward the adhesive-enamel interface. The micromechanical bond which connects the adhesive agent to the acid-etched enamel causes loss of enamel at adhesiveenamel interface due to adhesive failure. ${ }^{12}$

From the present study, the Transbond $\mathrm{XT}^{\prime}$ 's mean shear bond strength was shown to be $15.33 \mathrm{MPa}$; the findings are similar to that of Pickett et $\mathrm{al}^{13}$ and Arnold et $\mathrm{al}^{14}$ study, which showed Transbond XT's mean shear bond strength to be 9.7 MPa. Scougall Vilchis et al ${ }^{15}$ study, which compared the Transbond Plus with Transbond Plus and three other self-etching adhesives, showed that the Transbond XT's mean shear bond strength was highest of about 19.0 MPa compared with Transbond Plus (16.6 MPa) and other three adhesives. 
The Xeno V's mean shear bond strength from the present study was shown to be $17.46 \mathrm{MPa}$. Pithon et al ${ }^{16}$ compared and evaluated the conventional conditioner acid etchant with new self-etchant primer (Xeno V; seventh-generation bonding agent). There was no statistically significant difference in the shear bond strength of the Xeno group with Transbond group. The study conducted by Toledano et $\mathrm{al}_{1}{ }^{17}$ however, showed that Transbond XT had high shear bond strength compared with the Xeno V group, which is in contrast to the results from the present study.

The time-saving purpose not only applies for bonding purpose but also it interests the debonding, and removal of the residual resin from the tooth surface which are crucial for efficient and ideal workflow. More adhesive material attached to the bracket base, less time required, and the procedure will be safe and easy. One of the frequently used indexes to test adhesive material in orthodontics is ARI, which is a three-scaled quantifying scoring method for remnant adhesive introduced by Artun and Bergland. ${ }^{10}$

The ARI scores were higher under 0 and 1, except in group I, a score of 3 about $35 \%$ which is higher in number. This showed that the tested sample had a greater number of bond failures at adhesive-enamel interface which is consistent with the study results of Fleischmann et $\mathrm{al}^{18}$ and Lin et al. ${ }^{19}$ The low ARI scores of 0 and 1 are considered favorable from the Chang et $\mathrm{al}^{20}$ and Bishara et $\mathrm{al}^{21}$ study results, as there was less remnant adhesive on the enamel surface and consecutively less iatrogenic damage while enamel polishing. There are studies conducted on this topic, but the literature has conflicting results, to know whether low ARI scores are required or not.

In the bracket-adhesive-tooth system, the least failure is seen with metal bracket variable. Therefore, while accidental debonding of the brackets occurs during the treatment, the fracture appears at the enamel-composite interface. Generally, in clinical practice, the bonding system is chosen based on its adhesive property and handling procedure. ${ }^{22}$

The bracket bond strength can be enhanced clinically by altering the material and the bracket design which results in a homogeneous distribution of stress within the layer of cement during loading and minimizing the enamel damage during debonding of the orthodontic brackets. Therefore, this provides the guideline for the orthodontists during the selection of appropriate adhesive and orthodontic brackets for the patients' benefit. Reminder for the clinicians that this is a result of in vitro study, and the results may vary within oral environment. There is a need for further evaluation to investigate the influence of different adhesives and bracket base designs affecting stress distribution and bond strength of brackets used in orthodontics.

\section{CONCLUSION}

The study concluded that adhesive materials of the seventh generation yielded the shear bond strength values higher than that of the flowable composite and conventional acid etchant. The ARI scores indicated that there was a minimal amount of residual adhesive on the surface of the enamel after debonding.

\section{REFERENCES}

1. Russell JS. Aesthetic orthodontic brackets. J Orthod 2005 Jun;32(2):146-163.

2. Lavernhe $P$, Estivalèzes $E$, Lachaud $F$, Lodter $C$, Piquet R. Orthodontic bonding: finite element for standardized evaluations. Int J Adhes Adhes 2010 Jan;30(1):21-29.

3. Gioka C, Bourauel C, Zinelis S, Eliades T, Silikas N, Eliades G. Titanium orthodontic brackets: structure, composition, hardness and ionic release. Dent Mater 2004 Sep; 20(7):693-700.

4. Buonocore MG. A simple method of increasing the adhesion of acrylic filling materials to enamel surfaces. J Dent Res 1955 Dec;34(6):849-853.

5. Henkin FS, Macedo EO, Santos KS, Schwarzbach M, Samuel SM, Mundstock KS. In vitro analysis of shear bond strength and adhesive remnant index of different metal brackets. Dental Press J Orthod 2016 Nov-Dec;21(6):67-73.

6. Bishara SE, VonWald L, Laffoon JF, Warren JJ. Effect of a self-etch primer/adhesive on the shear bond strength of orthodontic brackets. Am J Orthod Dentofacial Orthop 2001 Jun;119(6):621-624.

7. Foersch M, Schuster C, Rahimi RK, Wehrbein H, Jacobs C. A new flash-free orthodontic adhesive system: a first clinical and stereomicroscopic study. Angle Orthod 2016 Mar;86(2):260-264.

8. Sachdeva A, Raghav S, Goel M, Raghav N, Tiwari S. A comparison of the shear bond strength of conventional acid etching, self-etching primer, and single bottle self-adhesivean in vitro study. Indian J Dent Sci 2017 Aug;9(3):170-175.

9. Kadam P, Bhalerao S. Sample size calculation. Int J Ayurveda Res 2010 Jan-Mar;1(1): 55-57.

10. Artun J, Bergland S. Clinical trials with crystal growth conditioning as an alternative to acid-etch enamel pretreatment. Am J Orthod 1984 Apr;85(4):333-340.

11. Mizrahi E, Smith DC. The use of cyanoacrylate adhesives for bonding orthodontic attachments. J Dent Res 1967;46: 1425-1432.

12. Karim Soltani M, Barkhori S, Alizadeh Y, Golfeshan F. Comparison of debonding characteristics of the conventional metal and self-ligating brackets to enamel: an in vitro study. Iran J Orthod 2014Aug;9(3):e4842.

13. Pickett KL, Sadowsky PL, Jacobsen A, Lacefield W. Orthodontic in vivo bond strength: comparison with in vitro results. Angle Orthod 2001 Apr;71(2):141-148.

14. Arnold RW, Combe EC, Warford JH. Bonding of stainless steel brackets to enamel with a new self-etching primer. Am J Orthod Dentofacial Orthop 2002 Sep;122(3):274-276.

15. Scougall Vilchis RJ, Yamamoto S, Kitai N, Yamamoto K. Shear bond strength of orthodontic brackets bonded with different self-etching adhesives. Am J Orthod Dentofacial Orthop 2009 Sep;136(3):425-430. 
16. Pithon MM, dos Santos RL, Ruellas AC, Sant'Anna EF. Onecomponent self-etching primer: a seventh generation of orthodontic bonding system? Eur J Orthod 2010 Oct;32(5): 567-570.

17. Toledano M, Osorio R, Osorio E, Romeo A, de la Higuera B, García-Godoy F. Bond strength of orthodontic brackets using different light and self-curing cements. Angle Orthod 2003 Feb;73(1):56-63.

18. Fleischmann LA, Sobral MC, Santos Júnior GC, Habib F. Comparative study of six types of orthodontic brackets for adhesion strength. Rev Dent Press Ortod Ortop Facial 2008 Jul-Aug;13(4):107-116.

19. Lin CL, Huang SF, Tsai HC, Chang WJ. Finite element submodeling analyses of damage to enamel at the incisor enamel/ adhesive interface upon de-bonding for different orthodontic bracket bases. J Biomech 2011 Jan;44(1):134-142.

20. Chang WG, Lim BS, Yoon TH, Lee YK, Kim CW. Effects of salicylic-lactic acid conditioner on the shear bond strength of brackets and enamel surfaces. J Oral Rehabil 2005 Apr;32(4): 287-295.

21. Bishara SE, VonWald L, Olsen ME, Laffoon JF. Effect of time on the shear bond strength of glass ionomer and composite orthodontic adhesives. Am J Orthod Dentofacial Orthop1999 Dec;116(6):616-620.

22. Valletta R, Prisco D, De Santis R, Ambrosio L, Martina R. Evaluation of the debonding strength of orthodontic brackets using three different bonding systems. Eur J Orthod 2007 Dec;29(6):571-577. 